MATEC Web of Conferences 32, 01002 (2015)

DOI: $10.1051 /$ matecconf $/ 20153201002$

(c) Owned by the authors, published by EDP Sciences, 2015

\title{
2D MEMS electrostatic cantilever waveguide scanner for potential image display application
}

\author{
Kebin Gu ${ }^{1, a}$, Keng-Ren Lin ${ }^{4}$ and Wei-Chih Wang ${ }^{1,2,3,4 a}$ \\ ${ }^{1}$ Department of Mechanical Engineering, University of Washington, Seattle, Washington, USA \\ ${ }^{2}$ Department of Electrical Engineering, University of Washington, Seattle, Washington, USA \\ ${ }^{3}$ Medical Device Innovation Center at National Cheng-Kung University, Tainan, Taiwan, R.O.C. \\ ${ }^{4}$ Department of BioMedical Engineering, National Cheng Kung University, Tainan, Taiwan, R.O.C.
}

\begin{abstract}
This paper presents the current status of our micro-fabricated SU-8 2D electrostatic cantilever waveguide scanner. The current design utilizes a monolithically integrated electrostatic push-pull actuator. A $4.0 \mu \mathrm{m} \mathrm{SU}-8$ rib waveguide design allows a relatively large core cross section $(4 \mu \mathrm{m}$ in height and $20 \mu \mathrm{m}$ in width) to couple with existing optical fiber and a broad band single mode operation $(\lambda=0.7 \mu \mathrm{m}$ to $1.3 \mu \mathrm{m})$ with minimal transmission loss ( $85 \%$ to $87 \%$ output transmission efficiency with Gaussian beam profile input). A 2D scanning motion has been successfully demonstrated with two fundamental resonances found at 202 and $536 \mathrm{~Hz}$ in vertical and horizontal directions. A $130 \mu \mathrm{m}$ and $19 \mu \mathrm{m}$, corresponding displacement and 0.062 and 0.009 rad field of view were observed at $\mathrm{a}+150 \mathrm{~V}$ input. Beam divergence from the waveguide was corrected by a focusing GRIN lens and a $5 \mu \mathrm{m}$ beam diameter is observed at the focal plane. The transmission efficiency is low $(\sim 10 \%)$ and cantilever is slightly under tensile residual stress due to inherent imperfection in the process and tooling in fabrication. However, 2D light scanning pattern was successfully demonstrated using 1-D push-pull actuation.
\end{abstract}

\section{Introduction}

In recent years, miniature scanner technologies, including micro-electro-mechanical system (MEMS) scanners, have started to appear on the market. Miniature scanner technologies have been employed in 1) scanning confocal microscopy, a powerful optical imaging method that can achieve sub-cellular resolution in real time [1-4], 2) portable, lightweight, low-power, inexpensive projection video displays with high information content [5-9], and 3) near-eye virtual displays like head-mounted displays (HMD) [10-14]. Most miniature scanner technologies utilize MEMS scanner mirrors. However, this approach has many limitations. For instance, mirror-based scanners are impractical for large angle beam deflection because mirror scanners and grating deflectors must be significantly larger than the source light beam diameter to avoid beam clipping or adding diffraction. Reducing the diameter of a conventional display device reduces the possible number of pixels, and thus reduces the resolution and/or field of view (FOV) of the device.

Our research team has previously developed a MEMS-based 2D micro image display device that can potentially overcome the size limitations of mirror-based display systems while maintaining high image resolution and FOV. The optical scanner consists of a microfabricated waveguide that is electromechanically deflected by a $2 \mathrm{D}$ piezoelectric actuator $[15,16]$. While functional, the original scanner design has a relatively large footprint (each individual driving actuator is $20 \times 4.8 \times 0.6 \mathrm{~mm}^{3}$ ) and is difficult to replicate accurately. Although off-the-shelf PZT bimorph actuators provide ample display actuation, the original scanner is too large to be effective, and the assembly of the device is difficult, time intensive, and unlikely to provide identical systems. The scanner also produces inconsistent results after each reset due to the way that the system is assembled.
This paper presents our improved waveguide scanner system, using a fully integrated MEMS-based push-pull actuator. The new design has several advantages over the previous generation: 1) a shorter rigid device length makes the potential micro display more flexible, able to access channels with sharp bends without the risk of penetration into the surrounding tissue. 2) incorporation of the light source and scanning waveguide probe into an integrated system, further reduces the system size. 3) MEMS devices provide good signal to noise ratios and smaller power consumption; and batch processing lowers fabrication costs while providing scalable production with consistent device quality and behavior.

\section{Design and Operation Principle}

\subsection{Operation principle}

The design and operation principle of the proposed micro scanner has been discussed in our previous publication [17] and we present only a summary here. The design of the scanner uses a pair of "push-pull" actuators to drive a cantilever waveguide (Fig. 1). The backscattered light is captured by the same optical scanner and channelled to an output detector. The waveguide is made of a long, slender SU-8 structure that runs through the middle of the scanner. At the input end of the waveguide, a coupler couples the light from an optical fiber via a $U$ shape fiber groove to the cantilever waveguide. At the distal (output) end, a proof mass is attached to the waveguide to purposely reduce the tip displacement while maintains the same angular deflection. A rotating arm connects the waveguide to the actuators. All actuating pads are of rectangular shape, suspended with the distal ends anchored to the substrate and the middle of the pads connected to the rotating arm. In this paper, a parallel-

\footnotetext{
${ }^{\mathrm{a}}$ Corresponding author: abong@uw.edu
} 
plate electrostatic MEMS actuator is presented. Fig. 2 shows two typical mode shapes of the waveguide.

(a) Coupling light source

(b) SU-8 waveguide and rib

(a)

(c) SU-8 proof mass

(d) Extended bottom electrode

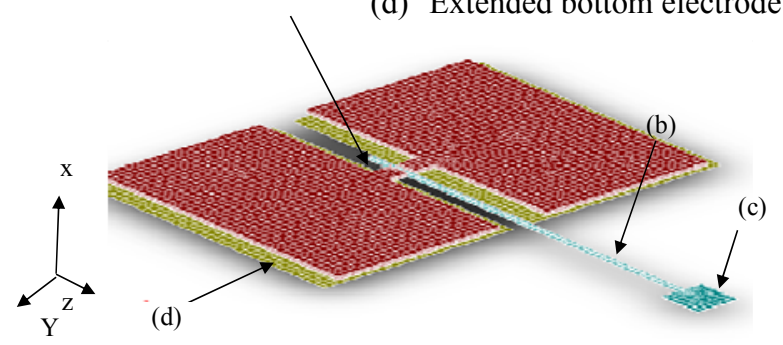

Figure 1. Schematic of the capacitive MEMS scanner using fringe effect for $\mathrm{Y}$ axis excitation enhancement.

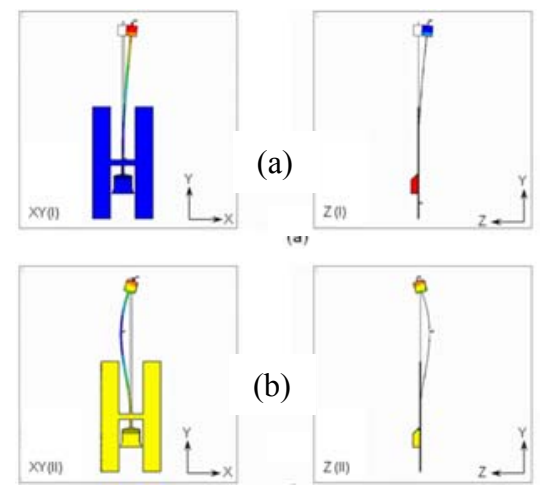

Figure 2. Typical mode shape of the waveguide.

A 2D raster or Lissajous rather than spiral motion is employed for the scanner. It is very difficult to create spiral motion with a nonsymmetrical mechanical beam profile (in this case a beam width that is $\sim 5$ times its overall thickness). It is also very difficult to fabricate a circular cladded waveguide using conventional microfabrication that utilizes a planar manufacturing process. Furthermore, a uniformly cladded waveguide will require a much larger cladding $(>50 \mu \mathrm{m})$ to confine the wave and maintains a single mode operation at the require geometry ( $\sim$ few $\mu \mathrm{m}$ for the core). Therefore, a rib waveguide design is chosen to allow a relatively small core (same size as the conventional optical fiber core) without no cladding for the scanner.

The basic operating principle of this 2-D scanner is shown in Fig. 3. The bending of the four actuating pads in or out of plane creates the necessary push or pull actuation. The resultant forces exerted by the four corners of the rotating arm can either move the arm along the waveguide direction or rotate the arm on the vertical plane, which in turn move the waveguide in or out of the plane. To generate oscillation in the horizontal plane, for example, pads 1 and 2 (or pads 3 and 4) must be moving in the same direction (in horizontal plane) and in phase
(Fig. 3a). This will cause the waveguide to move up or down in the vertical direction because of the hinge effect at the bottom layer of the coupler. To generate the bending in the horizontal plane, pads 1 and 3 (or 2 and 4) must be moving in the same direction with same magnitude and phase (Fig. 3b). This actuation is accompanied by an out-of-plane movement of the waveguide, due to the hinge effect at the bottom layer of the coupler. By exciting motion in the vertical and horizontal planes simultaneously, the tip of the waveguide will move in a raster/Lissajous scanning fashion. To achieve the scanning, the actuators must be driven by a signal formed by superimposing two carefully chosen resonant frequencies for the scanner system.
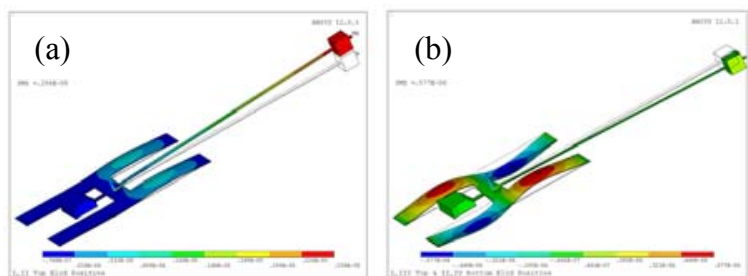

Figure 3. Basic operating principle of the 2-D scanner using push-pull actuator (a) showing displacement in vertical direction (b) showing beam deflection in horizontal plane.

\subsection{Design and Simulation}

The design and optimization process of the scanner design is performed by Architect3D in CoventorWare. Compared to traditional finite element method (FEM), the system-level model method can significantly shorten the simulation time with relatively high accuracy. Fig. 4 shows the schematic layout created in Architect3D and the corresponding 3D model. The schematic layout is composed of various components, which are used to model the mechanical and electrical behaviors of the scanner. The components include beams, beams with electrodes, rigid plate, anchors, bus connectors, reference frames and signal sources.

To optimize the performance of the scanner, two parameters, the scanning field of view (FOV) and the line resolution must be carefully examined. The high line resolution of the device is obtained through the large difference of the two operation frequencies. The resonant frequency of the high-frequency mode is designed to be 200 times larger than the resonant frequency of the lowfrequency mode. Therefore, highest scanning line resolution can be obtained. Since it is difficult to excite a device above $20 \mathrm{kHz}$ in our current experiment condition, the resonant frequency of the low-frequency mode must be smaller than $100 \mathrm{~Hz}$. As for the field of view, when the device is operating at its lower order mode resonant frequency, it results in larger FOV compared to higher order modes. 


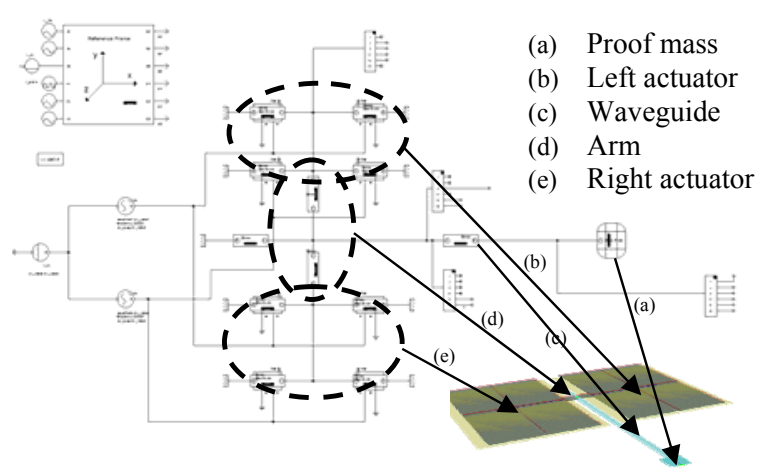

s.

Figure 4. Schematic of the scanner with illustrations of the $3 D$ physical geometry mapped to the corresponding behaviour symbols.

Because the frequency is a function of the mass and the spring constant, the low frequency can be achieved through a long waveguide or a large proof mass. However, a long waveguide undermines the device compactness. Therefore, we design the length of the waveguide and the size of proof mass as $2250 \mu \mathrm{m}$ and $300 \mu \mathrm{m}$, respectively. The modal analysis results obtained from Architect are verified by FEM results (Table 1). With the knowledge these preliminary results obtained from modal analysis, the optimization of the rotational angle of each mode is investigated next.

The excitation of vertical (low frequency) and horizontal motion (high frequency) using harmonic virtualization of the scanner in Architect3D under the electrostatic excitation is shown in Fig. 5. Fig. 6 shows the rotational angle response along both low and high frequency mode directions. In this direction, there are two resonance peaks. The designed low-frequency mode is chosen as $55.9 \mathrm{~Hz}$. The rotational angle is $0.44 \mathrm{rad}$. The middle trace is the rotational angle response along the high-frequency mode direction. The $12775 \mathrm{~Hz}$ peak is the high-frequency mode. The rotational angle is $0.089 \mathrm{rad}$. The bottom trace is the deflection response of the electrodes. At the high-frequency mode, the deflection of the electrodes is $4.27 \mu \mathrm{m}$ which is well below the designed gap space of $20 \mu \mathrm{m}$. The air damping is based on measuring value from a fiber viscometer [

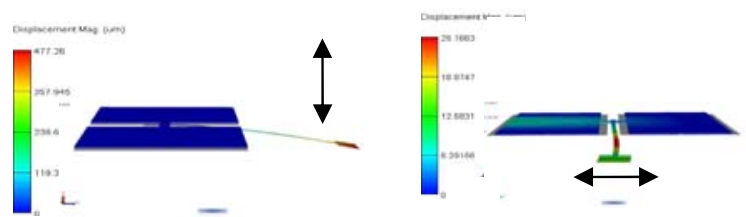

Figure 5. Harmonic virtualization of the scanner in Architect $3 D$ under the electrostatic excitation: (a) vertical motion and (b) horizontal motion.

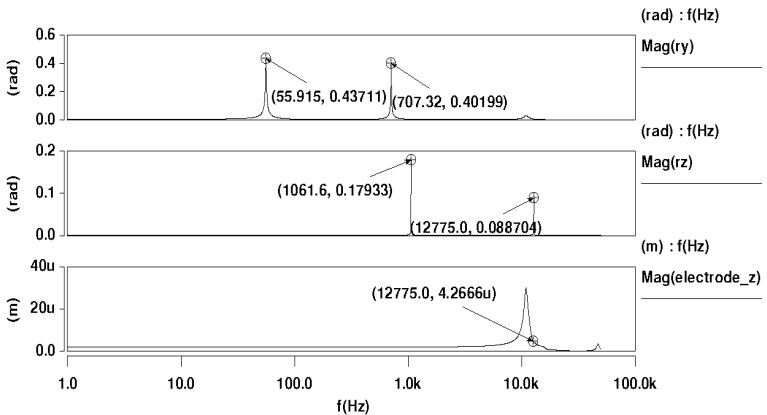

Figure 6. (Top trace): rotational angle response of the lowfrequency mode, (middle trace): rotational angle response of the low-frequency mode, and (bottom trace): deflection response of the electrodes.

Table 1. Resonant frequencies obtained by analytical estimation, FEM, and Architect3D.

\begin{tabular}{|c|c|c|c|c|}
\hline & & Analytical & FEM & Architect3D \\
\hline \multirow{2}{*}{-axis } & $1^{\text {st }}$ & 58.5 & 58.3 & 55.9 \\
\cline { 2 - 5 } & $2^{\text {nd }}$ & 743 & 630 & 707 \\
\hline Y-axis & $1^{\text {st }}$ & 1113 & 1103 & 1062 \\
\hline
\end{tabular}

\section{Fabrication}

\subsection{Scanner fabrication process}

The fabrication procedure of the electrostatic scanner is shown in Fig. 7. The device to be produced was fabricated from a 4 inch silicon wafer with a standard thickness of $525 \pm 25 \mu \mathrm{m}$. The substrate was cleaned by immersing a Piranha solution $\left(\mathrm{H}_{2} \mathrm{SO}_{4}: \mathrm{H}_{2} \mathrm{O}_{2}=4: 1\right)$ to clean organic residues off substrate. The wafer was then rinsed thoroughly in deionized (DI) water, dried using spin rinse dryer (SRD), and then dehydration on a hotplate at $200^{\circ} \mathrm{C}$ for 10 minutes. The processing begins with a two-layer SU-8 structures lithographic process. The first layer $(4 \mu \mathrm{m})$ of SU-8 5 (MicroChem, MA) was spun coat onto the silicon substrate to fabricate the micro display body and waveguide slab part, following instructions provided by the MicroChem Corp. To fabricate waveguide rib part, another $4 \mu \mathrm{m}$-thickness SU8 was spun and exposed on top of the first layer after post-exposure bake (PEB) process of the first SU-8 layer. In order to reduce the extrinsic stress that occurred during prebaking, the temperature was first holding at $70{ }^{\circ} \mathrm{C}$ for one minute before continuing ramping from $70{ }^{\circ} \mathrm{C}$ to $105^{\circ} \mathrm{C}$ at a rate of $3^{\circ} \mathrm{C} / \mathrm{min}$. We then held the sample at that temperature for 15 minutes before gradually ramping down to room temperature at a rate of $2^{\circ} \mathrm{C} / \mathrm{min}$.

Both waveguide slab and rib were transferred from soda lime masks to the double-layer SU-8 film by exposing the film on a contact aligner. For the PEB, the wafer was again placed on a hotplate for $1 \mathrm{~min}$. at $70^{\circ} \mathrm{C}$, holding for $1 \mathrm{~min}$, then ramping from $70^{\circ} \mathrm{C}$ to $105^{\circ} \mathrm{C}$ at a rate of $3^{\circ} \mathrm{C} / \mathrm{min}$, held the sample at that temperature for another 1 minute, gradually ramping down to room temperature for 30 minutes. This cooling period was longer than the post bake time recommended by the manufacturer. After cooling to room temperature, the double-layer SU-8 film was then developed in developer 
(PMGEA, an ethyl lactate and diacetone alcohol, MicroChem Corp., MA) for 1 minute with mild agitation. The wafer was then rinsed thoroughly in isopropyl alcohol (IPA) for another $1 \mathrm{~min}$., dried using SRD.

Metal electrodes made $20 \mathrm{~nm}$ Ti and $200 \mathrm{~nm}$ Au were done by an E-beam evaporation and lift-off process. Prior to metal deposition, a layer of negative photoresist NR93000PY (Futurrex Inc) was spun onto the SU-8 layer. The pattern was transferred from a soda lime mask to the resist to define electrodes area. Similar to previous process, subsequent photolithography and deep RIE, fiber groove was formed. Finally the device (scanner and bottom electrodes) were released via bulk silicon etching using deep RIE from the backside.

The fabrication process of the top electrode is a twostep micro-fabrication process. In a second 4 inch wafer, first the substrate was again followed the same clean and dry procedure as mentioned above. A photolithography process was followed to define the top electrodes area, an e-beam evaporator system is used again to deposit the Titanium adhesion layer and the Au electrode. The metal thin films were then patterned by lift-off process. Secondly a SU-8 spacer was spun and patterned to form a spacer. By the aid of an extra packaging design, which will be introduced in the next section, the top electrodes was being able to be aligned with the scanner and the bottom electrodes to be constructed with the parallelplates electrostatic actuation.

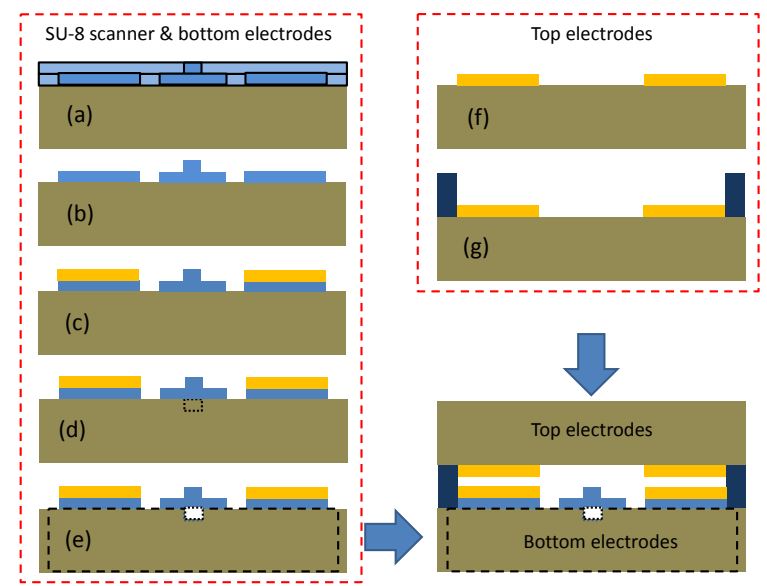

Figure 7. Fabrication process of electrostatic scanner. (a)-(e): scanner body and bottom electrodes (f)-(g): top electrodes. (a) Double-layer photolithography process to define rib shape waveguide and scanner pads (b) Development process to create SU-8 waveguide and body (c) Au/Ti thin films are deposited and patterned by lift-off process (d) Front side fiber grooves are patterned and deep etched using DRIE (e) the scanner (bottom electrode and waveguide) were released by DRIE again from the backside of the wafer (e) Backside etch-through to release actuators and waveguide (f) top electrode is deposited and patterned on a second silicon wafer ( $g$ ) SU-8 spacers are spun and patterned.

\subsection{Device package}

The packaging design is required in this study due to the fact that 1) the scanner needs to be durable enough to withstand situations similar to what the device will experience from contact with tissue under normal use, and 2) to secure the MEMS waveguide with an optical component, a focusing gradient index (GRIN) lens. Here an $8.0 \mathrm{~mm} \times 8.0 \mathrm{~mm} \times 1.0 \mathrm{~mm}$ holder made of acrylicbased photopolymer materials was designed and fabricated using a multi-material 3-dimensional printing system (Connex 350, Objet Geometries Ltd) with 16micrometer accuracy. The holder is made of 2 parts, and they are combined to form a ferrule to hold the GRIN lens at a predetermined distance from the tip of the cantilever waveguide as shown in Fig. 8. Our proposed GRIN lens is fixed and disposed beyond the end of the cantilever inside a proposed holder, relaying the image of the waveguide to an eventual object plane. Based on calculation, the angular deflection of the proposed scanning system is only 5 degree total (2.5 degree each side). The aberrations and vignette are minimal in such a small deflection. The $4.85 \mathrm{~mm}$ length $\times 2 \mathrm{~mm}$ diameter GRIN lens rod (GRINTECH GmbH, Jena, Germany) with working distance $=20 \mathrm{~mm}$, beam width $=20 \mu \mathrm{m}$, view angle $= \pm 30^{\circ}$, and $\mathrm{NA}=0.5$ was used to help steer and focus the diverging beam radiating out from the scanner. The estimated beam width at focus based on the given focal length of the grin lens and our operating wavelength is around 20 microns based on the $0.4 \mathrm{~mm}$ the gap space between the waveguide and grin lens design. The actual beam profile measurement is around 20 microns. The deviation is likely due to the larger gap space between grin lens and waveguide created by the inherent limitation on resolution of the $3 \mathrm{D}$ printer.

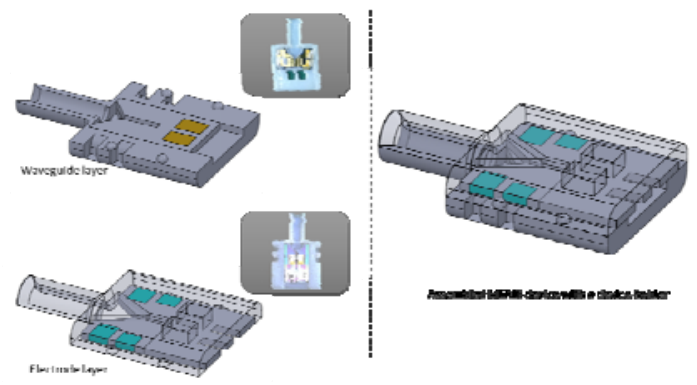

Figure 8. Schematics of assembles MEMS device including top electrode, scanner, bottom electrodes, and GRIN lens with the device holder.

\section{Results and Discussion}

The device was successfully fabricated (Figure 9a). However, due to its large aspect ratio and residual stresses, the waveguide appeared bent (Fig.9b). This problem has been resolved by carefully controlling the baking process of the SU-8 waveguide layers. Using more gradual heating and cooling resulted in a straighter beam (Fig. 9c). The new baking recipe also helped resolve the adhesion problem between the SU-8 waveguide and the silicon nitride substrate. In the earlier baking recipe, the SU-8 tended to peel off the nitride surface (Fig. 10a), because the thermal conductivity of nitride is much lower than silicon, which the recipe is based on. After a higher bake temperature is employed (originally $65^{\circ} \mathrm{C}$ for soft bake and $95^{\circ} \mathrm{C}$ for post-exposure bake, changed to $70^{\circ} \mathrm{C}$ and $105^{\circ} \mathrm{C}$, respectively), the delaminating problem is solved (Fig. 10b). 


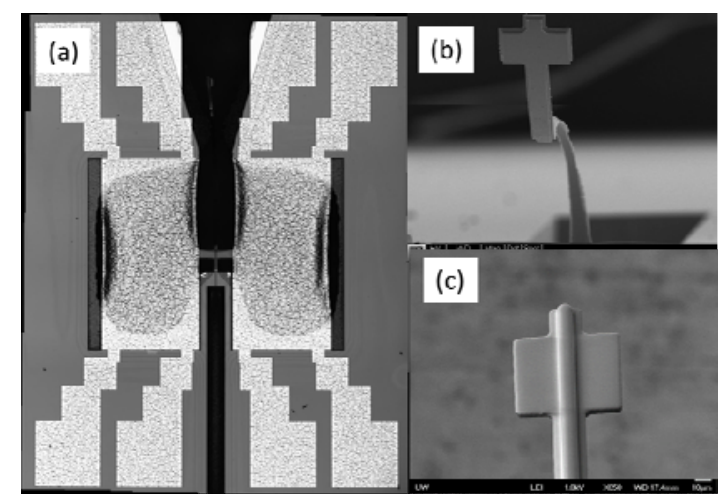

Figure 9. (a) Top view of the micro-fabricated MEMS scanner. (b), (c) SEM pictures of double-layer SU-8 waveguide.
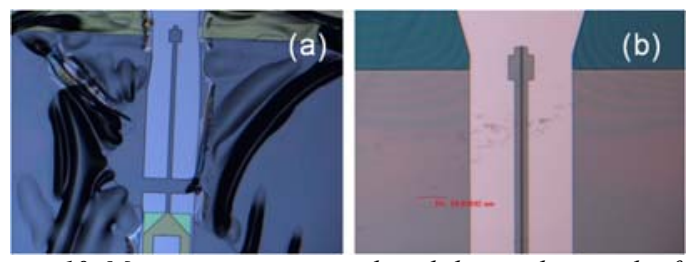

Figure 10. Microscopic pictures show lithographic result of double-layer SU-8 layer on top of nitride/silicon wafer. (a) Lower baking temperature $\left(65^{\circ} \mathrm{C} / 95^{\circ} \mathrm{C}\right)$ (b) Higher baking temperature $\left(70^{\circ} \mathrm{C} / 105^{\circ} \mathrm{C}\right)$.

When releasing the device using backside DRIE, the DRIE etch rate is not uniform. The center of the opening was released faster than the corner (Fig. 11). This causes an over-etch problem on the SU-8 waveguide when running more DRIE etch iterations to release the silicon residue around the corners. To solve this problem, the next prototype will use a revised mask design to protect the waveguide, or a slightly thicker SU-8 waveguide will be fabricated to compensate for over-etching.

The scanner test sample is prepared as shown in Fig. 12. Fig. 12 a shows the device test sample including both top and bottom electrodes and the RP holder with expended pads made of copper tapes sitting on a glass slide. An apparatus for the device on the glass slide is constructed to couple light into the fabricated SU-8 waveguide. The system uses an XYZ positioning stage for the light-carrying fiber to accurately couple to the SU8 waveguide. In order to observe the scanning motion of the waveguide tip, two cameras equipped with high power lenses (250× magnification) were set to provide aerial view and front view images of the SU- 8 waveguide tip (Fig. 12b). A $10 \mathrm{~mW} \mathrm{He-Ne} \mathrm{laser}(\lambda=632.8 \mathrm{~nm})$ provides the necessary light to the single mode optical fiber $($ diameter $=4.3 \mu \mathrm{m})$. Fig. 13 shows the aerial view of the waveguide with light coupled into the waveguide.

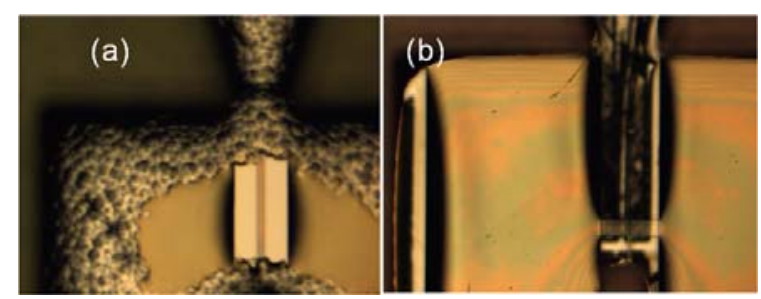

Figure 11. Backside view of (a) after 750 DRIE loops (b) add 30 more DRIE loops to clean the silicon residue around corner.

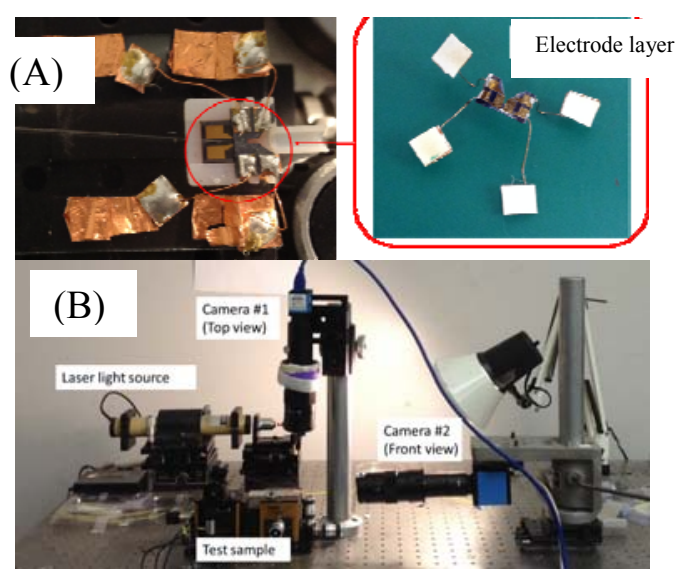

Figure 12. (a) MEMS device test sample (b) two cameras equipped with high power lenses $(250 \times$ magnification $)$ were set to provide aerial view and front view images.

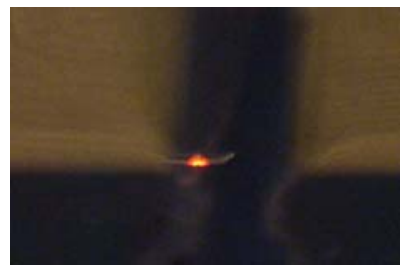

Figure 13. Aerial view of the waveguide with light coupled into the waveguide.

Several aspects of the performance of the device was tested. The vertical scan was achieved by applying $100 \mathrm{~V}$ $\mathrm{DC}$ voltage to the bottom electrodes and $\pm 150 \mathrm{~V}$ AC voltage to both of the top electrodes (left and right) with the same phase. The horizontal scan can also be observed by driving actuators with the same voltage as vertical scan but with 180 degree phase delay to top electrodes. The applied driving voltages are much larger than expected due to the fact that the gap space between top and bottom electrodes is larger than original designed value $(20 \mu \mathrm{m})$. The resonant frequencies for both directions were found by sweeping the frequency from 10 $\mathrm{Hz}$ to $600 \mathrm{~Hz}$ (Fig. 14). The resonant frequencies were found to be $\sim 201 \mathrm{~Hz}$ in the vertical direction, which is three times higher than the simulation result $(56 \mathrm{~Hz})$, and $\sim 535 \mathrm{~Hz}$ in the horizontal scan, about half of the simulation result $(1062 \mathrm{~Hz})$. As expected, the waveguide tip displacement in the horizontal direction is relatively small compare to the vertical direction $(19 \mu \mathrm{m}$ and 130 $\mu \mathrm{m}$, respectively, which is about 0.009 and $0.062 \mathrm{rad}$ as the field of view). Again the variation are most likely due to the fabrication imperfection, including the thickness variation of the SU-8 waveguides, and the plasma damage during the backside etch step. Fig. 15 presents the images of the characterization of the actuator membrane performance with static actuation versus applied DC voltages. The actuator membrane deflection is achieved by grounding bottom electrodes and applying voltage to the top electrodes. Visible deformation of the actuator membrane starts from the voltage $200 \mathrm{~V}$.

Characterization of the scanning performance of the waveguide are performed by measuring the waveguide tip displacement and scanning angle versus input actuation voltages (Fig. 16). The scanning angle is 
calculated as the inverse of the sine function of the tip displacement over the waveguide length, in this case, $2100 \mu \mathrm{m}$. Due to the large electric field necessary to actuate the actuators, an amplifier $(30 \times)$ must be used. Both tip displacement and scanning angle versus actuation voltages are highly linear. The largest test voltage is $150 \mathrm{~V}$, with a tip displacement of $111.8 \mu \mathrm{m}$ under $1 \mathrm{~Hz}$ driving frequency.

The initial displacement measurement of the scanner is smaller than expected due to smaller than expected actuation from the actuator. The probable cause of the smaller than expected actuation is likely due to the residual silicon thickness that was not completely removed during the release of the actuators (Fig. 17). This additional silicon layer most likely increases the stiffness and thus reduced the actuation.

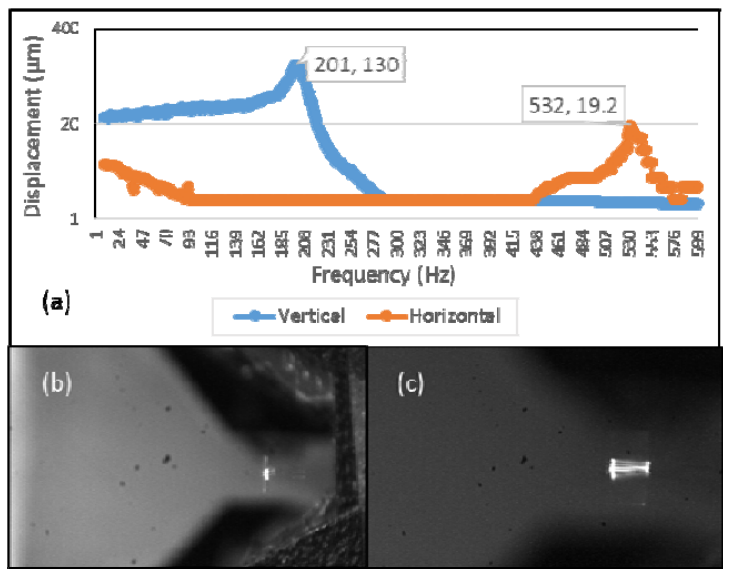

Figure 14. (a) Waveguide tip displacement vs. resonant frequency under the driving voltages with $100 \mathrm{~V}$ DC to bottom electrodes and $150 \mathrm{~V} A C$ to top electrodes, and microscope pictures show driving frequency under (b) $0 \mathrm{~Hz}$, (c) $201 \mathrm{~Hz}$.

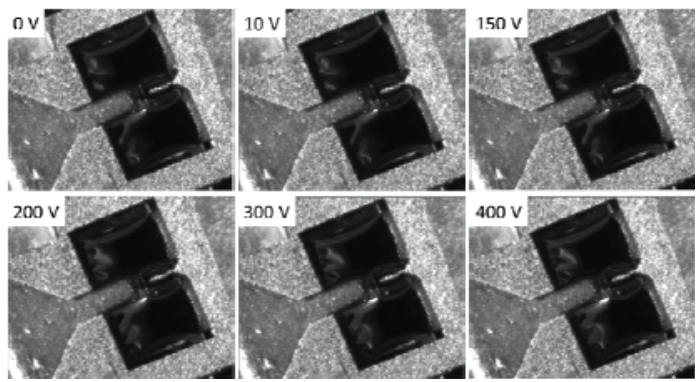

Figure 15. Statistic actuator deformation under various input DC voltage.

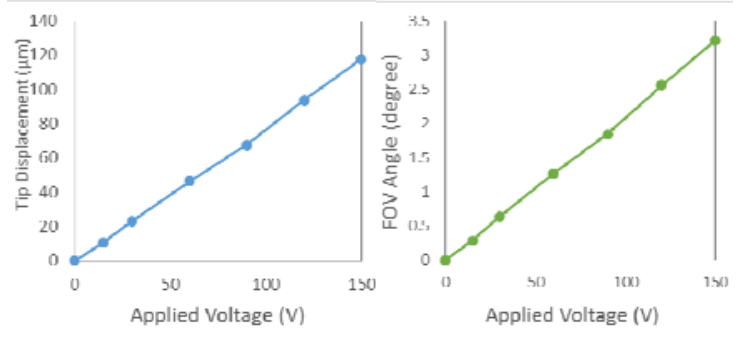

Figure 16. (a) Waveguide tip displacement and (b) scanning angle vs. varies applied voltage under $1 \mathrm{~Hz}$ driving frequency.

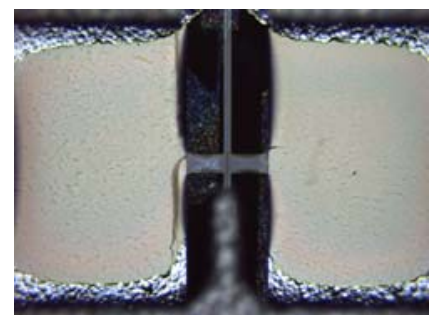

Figure 17. Microscopic picture shows residual silicon around corner of actuators after deep RIE released. Picture taken from the backside and focus on the surface of the actuators.

\section{Conclusion}

In this paper, we describe a new scanner design based on micro-fabrication technique to further reduce the size of a resonance-based optical scanner. This new scanning probe is constructed with a SU-8 waveguide actuated by a push-pull actuator, and is the first proposed MEMS scanner which integrates actuators into a resonance-based waveguide scanner.

The scanner's geometric parameters were analyzed to optimize the system's line resolution and FOV. Based on the simulation, the cross-section and length of the waveguide were found to be the dominant factors affecting scanner resolution; higher resolutions can be achieved with a vertically thin or horizontally wide inverted $\mathrm{T}$ cross section and a longer waveguide.

From the mechanical resonance test, we found the horizontal resonant of $536 \mathrm{~Hz}$ and vertical resonant of $201 \mathrm{~Hz}$. Deviations to the simulation results are most likely contributed to fabrication errors. With $10 \%$ deviation in the width and/or thickness, resonant frequency can vary up to greater than $10 \%$. The initial coupling tests show a relatively low coupling efficiency of $10 \%$. Based on optical simulation, this double-layer SU-8 rib waveguide design has a relatively large cross section ( $4 \mu \mathrm{m}$ in height and $20 \mu \mathrm{m}$ in width) which should provide very good coupling efficiency ( $95 \%$ with a Gaussian beam profile input) and a broad band single mode operation $(\lambda=0.7$ to $1.3 \mu \mathrm{m})$ with a minimal transmission loss $(0.85 \%$ output transmission efficiency). The discrepancy is likely due to the inherent process imperfection and tooling in creating the device and compounded by the micron range precision alignment needed to couple light from the fiber into the waveguide. For the FOV and line resolution test, the largest FOV is found to be $0.009 \mathrm{rad}$ in horizontal and $0.062 \mathrm{rad}$ in vertical $( \pm 150 \mathrm{~V})$ and the largest line resolution is calculated to be about 90 dots per line. Overall, the scanner has integrated electrostatic actuators into a resonance-based waveguide scanner. However, more revision in actuator and coupling system are needed to achieve a better FOV and line resolution.

\section{Acknowledgement}

The authors would like to thank Dr. Ryan Lee, Chi-Jung Lee, Chileung Tsui, and Chih-Hsuan Chien for their valuable comment and assistant in this project. This work is supported in part by grant R01EB007636-04. 


\section{References}

1. K. Murakami, A. Murata, T. Suga, H. Kitagawa, Y. Kamiya, M. Kubo, and M. Katashiro, TRANSDUCERS 1 587-590 (2003).

2. H. Ra, W. Piyawattanametha, Y. Taguchi, D. Lee, M. J. Mandella, and O. Solgaard, J. MEMS, 16, 969976 (2007)

3. H. Miyajima, N. Asaoka, T. Isokawa, M. Ogata, Y. Aoki, M. Imai, and K. Matsumoto, Microelectromechanical Systems, Journal of, 12(3), 243-251 (2003)

4. H. J. Shin, M. C. Pierce, D. Lee, H. Ra, O. Solgaard, and R. Richards-Kortum, Opt. Express, 15(15), 9113-9122 (2007)

5. Y. Hwang, J. Park, Y. Kim, and J. Kim, Proceedings of the 18th International Display Research Conference ASIA Display '98, pp. 1167-1170 (1998)

6. K. Yamada, T. Kuriyama, The 11th Annual International Workshop on Micro Electro Mechanical Systems, January 25-29, Heidelberg, Germany, pp. 110-115 (1998)

7. O. Solgaard, F. S. A. Sandejas, and D. M. Bloom, Optics letters, 17(9), 688-690 (1992)

8. D.T. Amm, and R.W. Corrigan, Society for Information Displays Symposium, Anaheim, CA, pp. 29-32 (1998)

9. A. Francis, M.-H. Kiang, O. Solgaard, and K.Y. Lau, Electron. Lett. 33, 1143-1145 (1997)

10. H. Urey, D. W. Wine, and T. D. Osborn, P SOC PHOTO-OPT INS, 176-185 (2000)

11. D. W. Wine, M. P. Helsel, L. Jenkins, H. Urey, T. D. Osborn, P SOC PHOTO-OPT INS, 186-196 (2000)

12. F. Helmchen, M. S. Fee, D. W. Tank, and W. Denk, Neuron, 31(6), 903-912. (2001)

13. R. S. Muller, and K. Y. Lau, Proceedings of the IEEE, 86(8), 1705-1720 (1998)

14. W. O. Davis, R. Sprague, and J. Miller, in Optical MEMs and Nanophotonics, IEEE/LEOS Internationall Conference on (pp. 31-32). IEEE. (2008)

15. W.-C. Wang, M. Fauver, J. N. Ho, E. J. Seibel, P. G. Reinhall, SENSOR ACTUAT A-PHYS 102, 165-175 (2002).

16. C. Takahshi, H. J. Wang, W. S. Hua, P. Reinhall, W. C. Wang, SPIE Health Monitoring and Smart Nondestructive Evaluation of Structural and Biological Systems, SPIE 6177 (2006).

17. K. Gu, C. L. Tsui, J. Ho, W. C. Wang, In SPIE Smart Structures and Materials+ Nondestructive Evaluation and Health Monitoring 83480T-83480T (2012)

18. W.Chih Wang, P. Reinhall, S. Yee, "Fluid viscosity measurement using forward light scattering [submerged optical fibre probe]", Measurement Science and Technology, 10 (4), 316-22 (1999) 